\title{
Cylindrical gear transmission optimization design based on fuzzy comprehensive evaluation
}

\author{
Guannan $\mathrm{HaO}^{1, \mathrm{a}}$
}

${ }^{1}$ Department of mechanical and electrical engineering, Binzhou University, 256600. P. R. China.

ahguannan@hotmail.com

Keywords: Cylindrical gear transmission, Optimization design, Fuzzy comprehensive evaluation

\begin{abstract}
In this paper, a fuzzy optimization method for gears has been proposed. The biggest advantages is to design the optimal solution under the existing conditions (including the level of equipment, technology, materials and design and manufacturing technology), and the proposed method was implemented by using the tool box of matlab, the results show that the fuzzy optimization method was greatly improved the efficiency and accuracy of design.
\end{abstract}

\section{Introduction}

Gear drive is used to change the size and the direction of the speed or torque (power)it was widely used in our industry[1]. The force in gear transmission is more complex, there are many uncertain factors from manufacturing to the actual operation[2], the value of the design parameters and allowable stress limitation, the level of design and manufacturing, materials, and the importance of parts have certain fuzziness[3], In the optimization design, if we consider the fuzziness of the various factors that will influence the design, the effect optimization will be improved obviously, in this paper the author proposed an idea that we can use fuzzy comprehensive evaluation for Cylindrical gear transmission optimization design, in this method, it consider fuzziness the of performance, the fuzzy optimization mathematical model is set up, he proposed method was implemented by using the tool box of matlab, the results show that the proposed was greatly improved the efficiency and accuracy of design.

\section{Design variables and Objective function}

In order to obtain transmission device with the smallest volume and lightest quality at the premise of the giving conditions and meeting the performance. We selected the $\mathrm{Z} 1, \mathrm{~m}$ and $\psi$ as the design variables[4]:

$$
x=\left[\begin{array}{lll}
m & Z_{1} & \psi
\end{array}\right]^{T}=\left[\begin{array}{lll}
x_{1} & x_{2} & x_{3}
\end{array}\right]^{T} .
$$

In this article, we select minimum value of the sum of volume of two gear as the objective function, then it can be written as follows[4]:

$$
f(x)=V_{1}+V_{2}=\frac{1}{4} \pi\left(1+u^{2}\right)\left(m z_{1}\right)^{3} \psi=\frac{1}{4} \pi\left(1+u^{2}\right)\left(x_{1} x_{2}\right)^{3} x_{3}
$$

\section{The cylindrical gear transmission optimization design constraints}

Design variable constraint.

(1) Modulus conditions: $5 \leq m \leq 15$.

(2)Teeth condition of the small one: $20 \leq z_{1} \leq 32$.

(3)Tooth width conditions: $0.5 \leq \psi \leq 1.2$.

Performance constraint.

Tooth surface contact fatigue limit:

$$
\sigma_{H}=Z_{E} Z_{H} \sqrt{\frac{2 K T_{1}}{d_{1}^{3} \psi}} \sqrt{\frac{u+1}{u}}=Z_{E} Z_{H} Z_{u} \sqrt{\frac{2 K T_{1}}{\left(m z_{1}\right)^{3} \psi}} \leq\left[\sigma_{H}\right]
$$


So tooth root bending fatigue strength constraints is:

$$
\sigma_{F 1}=\frac{2 K T_{1}}{m^{3} z_{1}^{2} \psi} Y_{F a 1} Y_{F s a 1} \leq\left[\sigma_{F}\right]_{1}, \quad \sigma_{F 2}=\frac{2 K T_{1}}{m^{3} z_{1}^{2} \psi} Y_{F a 2} Y_{F s a 2} \leq\left[\sigma_{F}\right]_{2}
$$

The range of design variable values as shown in table 1.

Table1 the lower and upper value of fuzzy constraint[5]

\begin{tabular}{c|c|c|c|c|c|c|c}
\hline \multicolumn{2}{c|}{ lower and upper } & {$\left[\sigma_{H}\right]$} & {$\left[\sigma_{F}\right]_{1}$} & {$\left[\sigma_{F}\right]_{2}$} & $m$ & $z_{1}$ & $\psi$ \\
\hline \multirow{2}{*}{$\begin{array}{c}\text { Upper } \\
\text { bound }\end{array}$} & upper & 1400 & 410 & 350 & 16 & 38 & 1.32 \\
\cline { 2 - 8 } & lower & 1260 & 369 & 315 & 12 & 28 & 1.1 \\
\hline \multirow{2}{*}{$\begin{array}{l}\text { Lower } \\
\text { bound }\end{array}$} & upper & & & & 8 & 22 & 0.55 \\
\cline { 2 - 8 } & lower & & & & 5 & 18 & 0.45 \\
\hline
\end{tabular}

Ordinary optimization constraints.

$$
\begin{aligned}
& g_{1}(x)=5-x_{1} \leq 0, g_{2}(x)=x_{1}-15 \leq 0, g_{3}(x)=20-x_{2} \leq 0, \\
& g_{4}(x)=x_{2}-32 \leq 0, g_{5}(x)=0.5-x_{3} \leq 0, g_{6}(x)=x_{3}-1.2 \leq 0, \\
& g_{7}(x)=\sigma_{H}-\left[\sigma_{H}\right]=Z_{E} Z_{H} Z_{u} \sqrt{\frac{2 K T_{1}}{\left(m z_{1}\right)^{3} \psi}}-\left[\sigma_{H}\right] \leq 0 \\
& g_{8}(x)=\sigma_{F 2}-\left[\sigma_{F}\right]_{1}=\frac{2 K T_{1}}{x_{1}^{3} x_{2}^{2} x_{3}} Y_{F a 2} Y_{F s a 2}-\left[\sigma_{F}\right]_{1} \leq 0, \\
& g_{9}(x)=\sigma_{F 1}-\left[\sigma_{F}\right]_{2}=\frac{2 K T_{1}}{x_{1}^{3} x_{2}^{2} x_{3}} Y_{F a 2} Y_{F s a 2}-\left[\sigma_{F}\right]_{2} \leq 0
\end{aligned}
$$

Fuzzy optimization constraints.

(1) Modulus conditions: $\underline{m} \leq m \leq \bar{m}$. (2) Teeth condition: $\underline{z_{1}} \leq z \leq \overline{z_{1}}$

(3)Tooth width conditions: $\underline{\psi} \leq \psi \leq \bar{\psi}$.

(4) Tooth surface contact fatigue strength limitation: $\sigma_{H}=\left[\sigma_{H}\right]$.

(5)The tooth root bending fatigue strength limitation: $\sigma_{F 1}=\left[\sigma_{F}\right]_{1}, \sigma_{F 2}=\left[\sigma_{F}\right]_{2}$.

The distribution of design variables is trapezoidal, Amplification coefficient method is adopted to define the boundary transition area, the upper and lower amplification coefficient of transition zone separately is $\bar{\beta}=1.1, \underline{\beta}=0.9$, design variables constraint cut sets is[3,4,5],Assume that distribution of performance constraints is drop half trapezoid,performance constraint cut sets is[6-7]. Therefore, constraint conditions of the fuzzy optimization is :

$$
\begin{aligned}
& g_{1}(x)=5+3 \lambda^{*}-x_{1} \leq 0, g_{2}(x)=x_{1}-\left(16-4 \lambda^{*}\right) \leq 0, g_{3}(x)=18+4 \lambda^{*}-x_{2} \leq 0, \\
& g_{4}(x)=x_{2}-\left(35-7 \lambda^{*}\right) \leq 0, g_{5}(x)=0.45+0.1 \lambda^{*}-x_{3} \leq 0, g_{6}(x)=x_{3}-\left(1.32-0.22 \lambda^{*}\right) \leq 0 \\
& g_{7}(x)=Z_{E} Z_{H} Z_{u} \sqrt{\frac{2 K T_{1}}{\left(m z_{1}\right)^{3} \psi}}-\left[1440-\lambda^{*}(1400-1260)\right] \leq 0 \\
& g_{8}(x)=\frac{2 K T_{1}}{x_{1}^{3} x_{2}^{2} X_{3}} Y_{F a 1} Y_{F s a 1}-\left[410-\lambda^{*}(410-369)\right] \leq 0 \\
& g_{9}(x)=\frac{2 K T_{1}}{x_{1}^{3} x_{2}^{2} x_{3}} Y_{F a 2} Y_{F s a 2}-\left[350-\lambda^{*}(350-315)\right] \leq 0
\end{aligned}
$$

\section{Cut sets level value $\lambda^{*}$}

According to value range of the cut sets element, the rally is 


$$
\lambda=\left[\begin{array}{llllllllll}
0.2 & 0.4 & 0.45 & 0.5 & 0.55 & 0.6 & 0.7 & 0.8 & 0.9 & 1.0
\end{array}\right]
$$

According to the selected factors that influence the design to determine the weight of various factors:

$$
A=\left[\begin{array}{llllll}
0.25 & 0.25 & 0.15 & 0.10 & 0.15 & 0.10
\end{array}\right]
$$

Factor levels and its membership degree shown in [5,6,7]. The single factor evaluation results are normalized processing.

$$
\omega_{i j}^{0}=\frac{R_{i j}^{0}}{\sum_{k=1}^{M} R_{i k}^{0}}
$$

Putting the single factor evaluation results together, we get fuzzy evaluation matrix:

$$
\underline{\omega^{0}}=\left[\begin{array}{ccccc}
0.3478 & 0.3913 & 0.2609 & 0 & 0 \\
0.3913 & 0.3478 & 0.2609 & 0 & 0 \\
0.3556 & 0.4222 & 0.2222 & 0 & 0 \\
0 & 0 & 0.2222 & 0.4222 & 0.3556 \\
0 & 0 & 0.1739 & 0.3913 & 0.4348 \\
0 & 0 & 0.1739 & 0.3913 & 0.4348
\end{array}\right]
$$

According to the cut sets step, determine judgment matrix of evaluation ranks of each factor ,assume that all factors evaluation's matrix have the same level,as follows:

$$
\underline{R^{1}}=\left[\begin{array}{cccccccccc}
1.0 & 0.95 & 0.8 & 0.5 & 0.2 & 0.1 & 0.0 & 0.0 & 0.0 & 0.0 \\
0.6 & 0.9 & 1.0 & 0.9 & 0.65 & 0.4 & 0.2 & 0.1 & 0.0 & 0.0 \\
0.3 & 0.5 & 0.65 & 0.85 & 1.0 & 0.9 & 0.6 & 0.4 & 0.2 & 0.1 \\
0.0 & 0.1 & 0.2 & 0.4 & 0.6 & 0.9 & 1.0 & 0.9 & 0.7 & 0.6 \\
0.0 & 0.0 & 0.1 & 0.2 & 0.4 & 0.7 & 0.9 & 0.95 & 0.97 & 1.0
\end{array}\right]
$$

Do the secondary fuzzy comprehensive evaluation by fuzzy matrix multiplication $B^{0}=w^{0} \circ R^{1}$.the secondary fuzzy comprehensive evaluation results is:

$$
\underline{B^{0}}=\left[\begin{array}{llllllllll}
0.6609 & 0.8130 & 0.8391 & 0.7478 & 0.5848 & 0.4261 & 0.2348 & 0.1435 & 0.0522 & 0.0261 \\
0.6783 & 0.8152 & 0.8304 & 0.7304 & 0.5652 & 0.4130 & 0.2261 & 0.1391 & 0.0522 & 0.026 \\
0.6756 & 0.8289 & 0.8511 & 0.7467 & 0.5678 & 0.4044 & 0.2178 & 0.1311 & 0.0444 & 0.0222 \\
0.0667 & 0.1533 & 0.2644 & 0.4289 & 0.6178 & 0.8289 & 0.8756 & 0.8067 & 0.6849 & 0.6311 \\
0.0522 & 0.1261 & 0.2348 & 0.3913 & 0.5826 & 0.8130 & 0.8870 & 0.8348 & 0.7304 & 0.6870 \\
0.0522 & 0.1261 & 0.2348 & 0.3913 & 0.5826 & 0.8130 & 0.8870 & 0.8348 & 0.7304 & 0.6870
\end{array}\right]
$$

The first level fuzzy comprehensive evaluation results is:

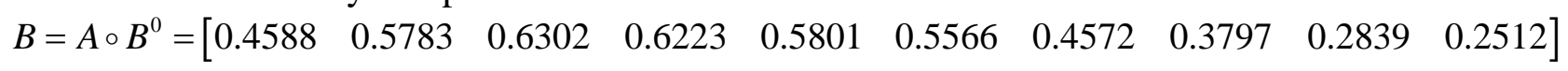

According to set the weighted average of cut set, cut sets level value is:

$$
\lambda^{*}=\frac{\sum_{i=1}^{L} \lambda_{i} \underline{B_{i}}}{\sum_{i=1}^{L} \underline{B_{i}}}, i=1,2, \ldots, L
$$

\section{The calculation example}

Transmission power $p=400 \mathrm{kw}$, speed $=550$, transmission ratio $u=3.43$.the small one's material is 22CrMnMo, tooth surface hardness after heat treatment $H R C=65$.For the big gear material is $12 \mathrm{CrMo}$, tooth surface hardness after heat treatment $H R C=58$. Contact fatigue strength 
$\left[\sigma_{H}\right]=1400 \mathrm{MPa}$ and bending fatigue strength of large and small gear, is respectively $\left[\sigma_{F}\right]_{2}=350 \mathrm{MPa}$ and $\left[\sigma_{F}\right]_{1}=410 \mathrm{MPa}$. Accordance to the proposed optimization method and the general optimization method, get the following results:

\begin{tabular}{|c|c|c|c|c|c|c|}
\hline \multirow{2}{*}{$\begin{array}{l}\text { Optimization } \\
\text { Method }\end{array}$} & \multicolumn{2}{|c|}{ modulus } & \multicolumn{2}{|c|}{$\mathrm{Z} 1$} & \multirow[b]{2}{*}{$\psi$} & \multirow{2}{*}{$\begin{array}{l}\text { Objective } \\
\text { function } \\
\text { value }\end{array}$} \\
\hline & Computation & Modification & Computation & Modification & & \\
\hline $\begin{array}{c}\text { General } \\
\text { optimization }\end{array}$ & 9.4 & 10 & 20 & 20 & 0.63 & $4.2151 * \mathrm{E} 7$ \\
\hline $\begin{array}{c}\text { Proposed } \\
\text { optimization } \\
\text { method }\end{array}$ & 7.54 & 8 & 20.25 & 21 & 1.196 & $4.2626 * \mathrm{E} 7$ \\
\hline
\end{tabular}

\section{Conclusions}

In this paper the author proposed a fuzzy optimization method for gears, Fuzzy optimization method for mechanical design, the biggest advantages is to design the optimal solution under the existing conditions (including the level of equipment, technology, materials and design and manufacturing technology), in another word: it Consider fuzziness the of performance, the fuzzy optimization mathematical model is set up. and the proposed method was implemented by using the tool box of matlab, the results show that the proposed Was greatly improved the efficiency and accuracy of design.

\section{References}

[1] X.Y. Du, X.L. Wang, Q.P. Yan. Finite Element Modeling and Analysis of Involute

Cylindrical Gear based on Matlab and UG. Journal of Mechanical Transmission,2011,36(7).

[2] G.H. Zhang, X.L. Zhu, R.S. Zeng. China. Stress Analyze of Medium Belt in Gear Transmission With Meshed Medium Belt. Journal of Chongqing University(Natural Science Edition),27 (2004) $1-5$.

[3] X.H. Zhang. Fuzzy Optimization Design of Cylinder Gear Based on Matlab. Coal Technology, 25 (2006) 17-20.

[4] W.B. Wang. The optimization design and mechanical parts. Beijing: mechanical industry publishing house, 1990.

[5] J.J. Meng. Fuzzy comprehensive assessment of cam moving rule in high speed warp knitting machine. Journal of Textile Research, 29 (2008) .

[6] Z.G. Liang, J.W. Shao, D.W. Sun. Research of Area Water-saving Irrigation Based on Fuzzy Comprehensive Evaluation.Water Conservancy Science and Technology and Economy, 17 (2011) 55-60.

[7] J. Ren, J. Li. Application of fuzzy comprehensive evaluation in route planning of highway passenger transportation. Journal of Chang'an University, 26 (2006) 83-86. 